\title{
On the directional derivative and directional continuity of set valued maps
}

\author{
Erdal Ekici
}




\title{
ON THE DIRECTIONAL DERIVATIVE AND DIRECTIONAL CONTINUITY OF SET VALUED MAPS
}

\author{
ERDAL EKICI
}

[Received: June 16, 2004]

\begin{abstract}
Aвstract. In this study, the directional lower and upper derivative sets of the set valued map the values of which have piecewise smooth boundary are investigated and obtained. Moreover, the connection between the directional continuity and the directional derivative sets of set-valued maps is investigated.
\end{abstract}

Mathematics Subject Classification: 26E25, 49J53, 58C20

Keywords: set-valued map, derivative sets, directional continuity

\section{InTROduction}

The concepts of the directional lower and upper derivative sets of set valued maps and continuity of set-valued maps are studied in many papers (see, e. g., [2,3, 5-7,9, $12-21])$.

The concepts of the directional lower and upper derivative sets of the set valued maps are based on the concepts of the lower and upper Bouligand cones [5,6]. In this paper, when the value of set-valued map has a piecewise smooth boundary, the directional lower and upper derivative sets are investigated. Furthermore, the connection between the directional continuity and the directional derivative sets of the set-valued maps is investigated.

In what follows, $\operatorname{cl}\left(\mathbb{R}^{m}\right)$ (resp., comp $\left.\left(\mathbb{R}^{m}\right)\right)$ denotes the set of all nonempty closed (resp., compact) subsets in $\mathbb{R}^{m}$.

Let $a(\cdot): \mathbb{R}^{n} \rightarrow \mathbb{R}^{m}$ be a set valued map and let $x_{0} \in \mathbb{R}^{n}$. It is said that $a(\cdot)$ is upper semi-continuous at $x_{0}$ if for all open neighbourhoods $N\left(a\left(x_{0}\right)\right)$ of the set $a\left(x_{0}\right)$, there exists a neighbourhood $N\left(x_{0}\right)$ of $x_{0}$ such that $a(x) \subset N\left(a\left(x_{0}\right)\right)$ for all $x \in N\left(x_{0}\right)$.

It is said that $a(\cdot)$ is lower semi-continuous at $x_{0}$ if for all $y_{0} \in a\left(x_{0}\right)$ and for all open neighbourhoods $N\left(y_{0}\right)$ of $y_{0}$, there exists a neighbourhood $N\left(x_{0}\right)$ of $x_{0}$ such that $a(x) \cap N\left(y_{0}\right) \neq \varnothing$ for all $x \in N\left(x_{0}\right)[3,18]$.

It is well-known that a set-valued map $a(\cdot): \mathbb{R}^{n} \rightarrow \operatorname{comp}\left(\mathbb{R}^{m}\right)$ is upper semicontinuous at $x_{0}$ if and only if for all $\varepsilon>0$, there exists a $\delta\left(\varepsilon, x_{0}\right)>0$ such that $\left\|x-x_{0}\right\|<\delta\left(\varepsilon, x_{0}\right) \Rightarrow a(x) \subset a\left(x_{0}\right)+\varepsilon B$, where $B=\left\{y \in \mathbb{R}^{m}:\|y\|<1\right\}$. 
Furthermore, $a(\cdot): \mathbb{R}^{n} \rightarrow \operatorname{comp}\left(\mathbb{R}^{m}\right)$ is lower semi-continuous at $x_{0}$ if and only if for all $\varepsilon>0$, there exists a $\delta\left(\varepsilon, x_{0}\right)>0$ such that $\left\|x-x_{0}\right\|<\delta\left(\varepsilon, x_{0}\right) \Rightarrow a\left(x_{0}\right) \subset$ $a(x)+\varepsilon B$, where $B=\left\{y \in \mathbb{R}^{m}:\|y\|<1\right\}[1,4]$.

It is said that the set-valued map $a(\cdot): \mathbb{R}^{n} \rightarrow \mathbb{R}^{m}$ is locally bounded at $x_{0} \in \mathbb{R}^{n}$ if $a(\cdot)$ is bounded in a neighbourhood of $x_{0}$.

\section{Directional DERIVATIVE SETS OF SET-VALUED MAPS}

Let $a(\cdot): \mathbb{R}^{n} \rightarrow \operatorname{cl}\left(\mathbb{R}^{m}\right)$ be an upper semi-continuous set-valued map. Let us consider the following sets. For $(x, y) \in \mathbb{R}^{n} \times \mathbb{R}^{m}$ and vector $f \in \mathbb{R}^{n}$, we set

$$
\begin{aligned}
& D a(x, y) \mid(f)=\left\{d \in \mathbb{R}^{m}: \liminf _{\delta \rightarrow+0} \frac{1}{\delta} \operatorname{dist}(y+\delta d, a(x+\delta f))=0\right\}, \\
& D^{*} a(x, y) \mid(f)=\left\{v \in \mathbb{R}^{m}: \lim _{\delta \rightarrow+0} \frac{1}{\delta} \operatorname{dist}(y+\delta d, a(x+\delta f))=0\right\} .
\end{aligned}
$$

Here for $x \in \mathbb{R}^{n}, D \subset \mathbb{R}^{n}, \operatorname{dist}(x, D)=\inf _{d \in D}\|x-d\| . D a(x, y) \mid(f)\left(D^{*} a(x, y) \mid(f)\right)$ is called the upper (lower) derivative set of the set valued map $a(\cdot)$ at $(x, y)$ in the direction $f$.

Note that the directional upper (lower) derivative set of the set-valued map $a(\cdot)$ is closed and there is a connection between the upper (lower) derivative set of a setvalued map and the upper (lower) contingent cone which is used to investigate several problems in nonsmooth analysis (see, e. g., $[3,8,15])$.

It is obvious that $D^{*} a(x, y)|(f) \subset D a(x, y)|(f)$.

$$
A=\operatorname{graph} a(\cdot)=\left\{(x, y) \in \mathbb{R}^{n} \times \mathbb{R}^{m}: y \in a(x)\right\}
$$

denotes the graph of the set-valued map $a(\cdot)$. Since $a(\cdot)$ is upper semicontinuous, graph $a(\cdot)$ is a closed set. It is possible to show that $D a(x, y)\left|(f)=D^{*} a(x, y)\right|(f)=$ $\varnothing$ if $(x, y) \notin \operatorname{graph} a(\cdot), D a(x, y)\left|(f)=D^{*} a(x, y)\right|(f)=\mathbb{R}^{m}$ if $(x, y) \in \operatorname{int}(\operatorname{graph} a(\cdot))$ where int (graph $a(\cdot)$ ) denotes the interior of graph $a(\cdot)$.

Suppose that the set-valued map $a(\cdot)$ is given as

$$
a(x)=\left\{y \in \mathbb{R}^{m}: c(x, y) \geq 0\right\}
$$

where $c(\cdot, \cdot): \mathbb{R}^{n} \times \mathbb{R}^{m} \rightarrow R$ is a continuous function on $\mathbb{R}^{n} \times \mathbb{R}^{m}$ and locally Lipschitz on $\mathbb{R}^{m}$. The lower and upper derivative of $c(\cdot, \cdot)$ at the point $(x, y)$ in the direction $(f, d)$ is denoted by $\frac{\partial^{-} c(x, y)}{\partial(f, d)}$ and $\frac{\partial^{+} c(x, y)}{\partial(f, d)}$, respectively, and defined by

$$
\begin{gathered}
\frac{\partial^{-} c(x, y)}{\partial(f, d)}=\liminf _{\delta \rightarrow+0}[c(x+\delta f, y+\delta d)-c(x, y)] \delta^{-1}, \\
\frac{\partial^{+} c(x, y)}{\partial(f, d)}=\limsup _{\delta \rightarrow+0}[c(x+\delta f, y+\delta d)-c(x, y)] \delta^{-1},
\end{gathered}
$$


respectively. If

$$
\frac{\partial c(x, y)}{\partial(f, d)}=\lim _{\delta \rightarrow+0}[c(x+\delta f, y+\delta d)-c(x, y)] \delta^{-1}
$$

exists and is finite, then $c(\cdot, \cdot)$ is called differentiable at the point $(x, y)$ in the direction $(f, d)$ and $\frac{\partial c(x, y)}{\partial(f, d)}$ denotes the derivative of $c(\cdot, \cdot)$ at the point $(x, y)$ in the direction $(f, d)$.

We introduce the sets

$$
\begin{aligned}
& H_{*}^{-}(x, y) \mid(f)=\left\{d \in \mathbb{R}^{m}: \frac{\partial^{+} c(x, y)}{\partial(f, d)}>0\right\}, \\
& H_{*}(x, y) \mid(f)=\left\{d \in \mathbb{R}^{m}: \frac{\partial^{+} c(x, y)}{\partial(f, d)} \geq 0\right\}, \\
& E_{*}^{-}(x, y) \mid(f)=\left\{d \in \mathbb{R}^{m}: \frac{\partial^{-} c(x, y)}{\partial(f, d)}>0\right\}, \\
& E_{*}(x, y) \mid(f)=\left\{d \in \mathbb{R}^{m}: \frac{\partial^{-} c(x, y)}{\partial(f, d)} \geq 0\right\} .
\end{aligned}
$$

Proposition 1. Let the set-valued map a(.) be in the form (2.1). Then for all $(x, y) \in \partial A$ and $f \in \mathbb{R}^{n}$,

$$
\begin{aligned}
& \operatorname{cl} H_{*}^{-}(x, y)|(f) \subset D a(x, y)|(f) \subset H_{*}(x, y) \mid(f), \\
& \operatorname{cl} E_{*}^{-}(x, y)\left|(f) \subset D^{*} a(x, y)\right|(f) \subset E_{*}(x, y) \mid(f),
\end{aligned}
$$

where $\partial A$ denotes the boudary of $A$ and $\mathrm{cl} A$ denotes the closure of $A$.

Proposition 2. Let $(x, y) \in \partial A, c(\cdot, \cdot)$ be differentiable at $(x, y)$ and $\frac{\partial c(x, y)}{\partial y} \neq 0$. Then it is possible to show that

$$
\begin{aligned}
\operatorname{Da}(x, y) \mid(f) & =D^{*} a(x, y) \mid(f) \\
& =\left\{d \in \mathbb{R}^{m}:\left\langle\frac{\partial c(x, y)}{\partial x}, f\right\rangle+\left\langle\frac{\partial c(x, y)}{\partial y}, d\right\rangle \geq 0\right\},
\end{aligned}
$$

where the symbol $\langle\cdot, \cdot\rangle$ denotes the inner product.

Remark 1 . Now suppose that the set-valued map $a(\cdot): \mathbb{R}^{n} \rightarrow \operatorname{cl}\left(\mathbb{R}^{m}\right)$ is given by the relation

$$
a(x)=\left\{y \in \mathbb{R}^{m}: \max _{i \in I} c_{i}(x, y) \geq 0\right\}
$$

where $I$ is a finite set and $c_{i}(\cdot, \cdot)$ is a continuous differentiable function for all $i \in I$. Then (see [10,11]) $b(x, y)=\max _{i \in I} c_{i}(x, y)$ is a directional derivable function and

$$
\frac{\partial c(x, y)}{\partial(f, d)}=\max _{i \in I_{*}(x, y)}\left[\left\langle\frac{\partial c_{i}(x, y)}{\partial x}, f\right\rangle+\left\langle\frac{\partial c_{i}(x, y)}{\partial y}, d\right\rangle\right],
$$

where

$$
I_{*}(x, y)=\left\{i_{*} \in I: c_{i_{*}}(x, y)=\max _{i \in I} c_{i}(x, y)\right\}
$$


In that case, it follows herefrom that

$$
\begin{aligned}
E_{*}^{-}(x, y) \mid(f) & =H_{*}^{-}(x, y) \mid(f) \\
& =\left\{d \in \mathbb{R}^{m}: \max _{i \in I_{*}(x, y)}\left[\left\langle\frac{\partial c_{i}(x, y)}{\partial x}, f\right\rangle+\left\langle\frac{\partial c_{i}(x, y)}{\partial y}, d\right\rangle\right]>0\right\}, \\
E_{*}(x, y) \mid(f) & =H_{*}(x, y) \mid(f) \\
& =\left\{d \in \mathbb{R}^{m}: \max _{i \in I_{*}(x, y)}\left[\left\langle\frac{\partial c_{i}(x, y)}{\partial x}, f\right\rangle+\left\langle\frac{\partial c_{i}(x, y)}{\partial y}, d\right\rangle\right] \geq 0\right\} .
\end{aligned}
$$

Theorem 1. Let the set-valued map $a(\cdot): \mathbb{R}^{n} \rightarrow \operatorname{cl}\left(\mathbb{R}^{m}\right)$ be of form $(2.2),(x, y) \in$ $\partial A, f \in \mathbb{R}^{n}$ and $H_{*}^{-}(x, y) \mid(f) \neq \varnothing$. Then

$$
\operatorname{Da}(x, y)\left|(f)=D^{*} a(x, y)\right|(f)=H_{*}(x, y) \mid(f) .
$$

Proof. It is obtained by using the preceding propositions and the remark.

Remark 2. The above theorem is not true when $H_{*}^{-}(x, y) \mid(f)=\varnothing$ for $(x, y) \in \partial A$ and for $f \in \mathbb{R}^{n}$.

Example 1. Let us take the set-valued map $a(\cdot):[0,1] \rightarrow \operatorname{cl}\left(\mathbb{R}^{2}\right), x \mapsto a(x)=$ $\left\{\left(y_{1}, y_{2}\right) \in \mathbb{R}^{2}:-y_{1}^{2}-y_{2}^{2} \geq 0\right\}$. We know that $a(x)=\{(0,0)\}$ for all $x \in[0,1]$ and $b(\cdot, \cdot, \cdot):[0,1] \times \mathbb{R}^{2} \rightarrow \mathbb{R},\left(x, y_{1}, y_{2}\right) \mapsto b\left(x, y_{1}, y_{2}\right)=y_{1}^{2}+y_{2}^{2}$ is a differentiable function. Then we obtain $H_{*}(x, 0,0)\left|(1)=\mathbb{R}^{2}, H_{*}^{-}(x, 0,0)\right|(1)=\varnothing$ and $D a(x, 0,0) \mid$ $(1)=\{(0,0)\}$ for $(x, 0,0) \in \partial A$.

\section{Directional CONTINUity OF SET-VALUED MaPS}

Theorem 2. Let $a(\cdot): \mathbb{R}^{n} \rightarrow \operatorname{comp}\left(\mathbb{R}^{m}\right)$ be a set valued map. If graph $a(\cdot)$ is closed and $a(\cdot)$ is locally bounded at $x_{0} \in \mathbb{R}^{n}$, then a $(\cdot)$ is upper semi continuous at $x_{0}[4,18]$.

Theorem 3. Let $a(\cdot): \mathbb{R}^{n} \rightarrow \mathbb{R}^{m}$ be a set valued map and let $x_{0} \in \mathbb{R}^{n}$. a $(\cdot)$ be lower semi continuous at $x_{0}$ if and only if for all $\left\{x_{n}\right\}_{n=1}^{\infty}$ where $x_{n} \rightarrow x_{0}$ as $n \rightarrow \infty$ and for all $y_{0} \in a\left(x_{0}\right)$, there exists $a\left\{y_{n}\right\}_{n=1}^{\infty}$ such that $y_{n} \in a\left(x_{n}\right)$ and $y_{n} \rightarrow y_{0}$ as $n \rightarrow \infty[3]$.

Definition 1. Let $x_{0} \in \mathbb{R}^{n}, f \in \mathbb{R}^{n}$ and let $a(\cdot): \mathbb{R}^{n} \rightarrow \operatorname{comp}\left(\mathbb{R}^{m}\right)$ be a set-valued map. If

$$
\lim _{\delta \rightarrow 0+} a\left(x_{0}+\delta f\right)=a\left(x_{0}\right)
$$

then it is said that $a(\cdot)$ is continuous at $x_{0}$ in the direction $f$. If

$$
\lim _{\delta \rightarrow 0+} a(x+\delta f)=a(x)
$$

for all $x \in \mathbb{R}^{n}$, then it is said that $a(\cdot)$ is continuous on $\mathbb{R}^{n}$ in the $\operatorname{direction} f$. 
Proposition 3. Let $x_{0} \in \mathbb{R}^{n}, f \in \mathbb{R}^{n}$ and let $a(\cdot): \mathbb{R}^{n} \rightarrow \operatorname{comp}\left(\mathbb{R}^{m}\right)$ be a setvalued map. The set valued map $\varphi(\cdot): \mathbb{R} \rightarrow \operatorname{comp}\left(\mathbb{R}^{m}\right), \delta \mapsto \varphi(\delta)=a\left(x_{0}+\delta f\right)$, is continuous on the right at $\delta=0$ if and only if $a(\cdot)$ is continuous at $x_{0}$ in the direction $f$.

Proposition 4. Let $x_{0} \in \mathbb{R}^{n}, f \in \mathbb{R}^{n}$ and $a(\cdot): \mathbb{R}^{n} \rightarrow \operatorname{comp}\left(\mathbb{R}^{m}\right)$ be a set-valued map. If the set valued map a $(\cdot)$ is upper continuous (resp., lower continuous) at $x_{0}$, then the set-valued map $\varphi(\cdot): \mathbb{R} \rightarrow \operatorname{comp}\left(\mathbb{R}^{m}\right)$,

$$
\delta \longmapsto \varphi(\delta)=a\left(x_{0}+\delta f\right),
$$

is upper continuous (resp., lower continuous) at $\delta=0$.

Hence, if the set-valued map $a(\cdot)$ is continuous at $x_{0}$, then the set-valued map $\varphi(\cdot): \mathbb{R} \rightarrow \operatorname{comp}\left(\mathbb{R}^{m}\right), \delta \mapsto \varphi(\delta)=a\left(x_{0}+\delta f\right)$, is continuous at $\delta=0$.

Proof. Let $f \in \mathbb{R}^{n}$ and let $a(\cdot)$ be continuous at $x_{0}$. We will show that there exists a $\delta(\varepsilon)>0$ such that

$$
|\delta|<\delta(\varepsilon) \quad \Longrightarrow \quad \varrho_{H}(\varphi(\delta), \varphi(0))=\varrho_{H}\left(a\left(x_{0}+\delta f\right), a\left(x_{0}\right)\right)<\varepsilon
$$

for all $\varepsilon>0$, where $\varrho_{H}$ denotes Hausdorff distance. Then the set-valued map $\varphi(\cdot)$ will be continuous at $\delta=0$.

Let $\varepsilon>0$. Since the set-valued map $a(\cdot)$ is continuous at $x_{0}$, then there exists a $\delta_{1}>0$ such that

$$
\left\|x-x_{0}\right\|<\delta_{1} \quad \Longrightarrow \quad \varrho_{H}\left(a(x), a\left(x_{0}\right)\right)<\varepsilon .
$$

Put $\delta_{*}=\delta_{1}\|f\|^{-1}$. Then for all $\delta \in\left(-\delta_{*}, \delta_{*}\right)$,

$$
\left\|x_{0}+\delta f-x_{0}\right\|=|\delta|\|f\|<\|f\| \cdot \frac{\delta_{1}}{\|f\|}=\delta_{1} .
$$

It follows that from (3.1), for all $\delta \in\left(-\delta_{*}, \delta_{*}\right)$,

$$
\varrho_{H}\left(a\left(x_{0}+\delta f\right), a\left(x_{0}\right)\right)<\varepsilon .
$$

Hence, we obtain that the set-valued $\operatorname{map} \varphi(\cdot)$ is continuous at $\delta=0$.

Theorem 4. Let $x_{0} \in \mathbb{R}^{n}$ and let $a(\cdot): \mathbb{R}^{n} \rightarrow \operatorname{comp}\left(\mathbb{R}^{m}\right)$ be a set-valued map. If the set-valued map $a(\cdot)$ is continuous at $x_{0}$, then $a(\cdot)$ is continuous at $x_{0}$ in the direction $f$ for any $f \in \mathbb{R}^{n}$.

Proof. Since the set-valued map $a(\cdot)$ is continuous at $x_{0}$, then the set valued map $\varphi(\cdot)$ is continuous at $\delta=0$ from Proposition 4. From Proposition 3, the set-valued map $a(\cdot)$ is continuous at $x_{0}$ in the direction $f$ for any $f \in \mathbb{R}^{n}$.

Remark 3. The converse of the above theorem is not true. The following example will show this. 
Example 2. Let $f(\cdot, \cdot): \mathbb{R} \times \mathbb{R} \rightarrow \mathbb{R}$ be the function defined by

$$
(x, y) \longmapsto f(x, y)= \begin{cases}x y /\left(x^{4 / 3}+y^{6}\right) & \text { for } x \neq 0, y \neq 0, \\ 0 & \text { for } x=0, y=0 .\end{cases}
$$

We will show that $f(\cdot, \cdot)$ is continuous at $(0,0)$ in the direction $\left(f_{1}, f_{2}\right)$ where $f_{1} \neq 0$ or $f_{2} \neq 0$, but $f(\cdot, \cdot)$ is not continuous at $(0,0)$.

Let $\left(x_{0}, y_{0}\right)=(0,0)$. Let $\left(f_{1}, f_{2}\right) \in \mathbb{R} \times \mathbb{R}$ such that $f_{1} \neq 0$ or $f_{2} \neq 0$. Then

$$
\begin{aligned}
f\left(\left(x_{0}, y_{0}\right)+\delta\left(f_{1}, f_{2}\right)\right) & =f\left(x_{0}+\delta f_{1}, y_{0}+\delta f_{2}\right) \\
& =f\left(\delta f_{1}, \delta f_{2}\right) \\
& =\delta^{2} f_{1} f_{2} /\left(\delta^{4 / 3} f_{1}^{4 / 3}+\delta^{6} f_{2}^{6}\right) \\
& =\delta^{2 / 3} f_{1} f_{2} /\left(f_{1}^{4 / 3}+\delta^{4 \frac{2}{3}} f_{2}^{6}\right)
\end{aligned}
$$

and hence, as $\delta \rightarrow+0$,

$$
\frac{\delta^{2 / 3} f_{1} f_{2}}{f_{1}^{4 / 3}+\delta^{4 \frac{2}{3}} f_{2}^{6}} \rightarrow 0=f(0,0) .
$$

Therefore, $f(\cdot, \cdot)$ is continuous at $(0,0)$ in the direction $\left(f_{1}, f_{2}\right)$.

Take $\left\{\left(x_{n}, y_{n}\right)\right\}_{n=1}^{\infty}=\left\{\left(\delta_{n}, \delta_{n}^{1 / 3}\right)\right\}_{n=1}^{\infty}$, where $\delta_{n} \rightarrow 0$ as $n \rightarrow \infty$. Then $\left(x_{n}, y_{n}\right) \rightarrow$ $(0,0)$. Since

$$
f\left(x_{n}, y_{n}\right)=\frac{\delta_{n}^{4 / 3}}{\delta_{n}^{4 / 3}+\delta_{n}^{2}}=\frac{1}{1+\delta_{n}^{2 / 3}}
$$

and $\left(1+\delta_{n}^{2 / 3}\right)^{-1} \rightarrow 1$ as $n \rightarrow \infty$, then it follows that

$$
\lim _{(x, y) \rightarrow(0,0)} f(x, y) \neq f(0,0) .
$$

Hence, $f(\cdot, \cdot)$ is not continuous at $(0,0)$.

Theorem 5. If the set-valued map $a(\cdot): \mathbb{R}^{n} \rightarrow \operatorname{comp}\left(\mathbb{R}^{m}\right)$ is continuous at $x_{0}=$ $\left(x_{0}^{1}, x_{0}^{2}, \ldots, x_{0}^{n}\right)$ in all directions $f \in \mathbb{R}^{n}$, then the set-valued map

$$
x^{i} \longmapsto a\left(x_{0}^{1}, x_{0}^{2}, \ldots, x_{0}^{i-1}, x^{i}, x_{0}^{i+1}, \ldots, x_{0}^{n}\right)
$$

is continuous at $x_{0}^{i}$.

Proof. Let $f_{1}=(0,0, \ldots, 0,1,0, \ldots, 0)$ and $f_{2}=(0,0, \ldots, 0,-1,0, \ldots, 0)$ where 1 and -1 are the $i$ th components. Since the set-valued map $a(\cdot)$ is continuous at $x_{0}$ in all directions $f \in \mathbb{R}^{n}$, we see that

$$
\begin{aligned}
& \lim _{\delta \rightarrow 0+} a\left(x_{0}+\delta f_{1}\right)=a\left(x_{0}\right), \\
& \lim _{\delta \rightarrow 0+} a\left(x_{0}+\delta f_{2}\right)=a\left(x_{0}\right) .
\end{aligned}
$$


Then we obtain

$$
\begin{aligned}
& \lim _{\delta \rightarrow 0+} a\left(x_{0}^{1}, x_{0}^{2}, \ldots, x_{0}^{i-1}, x_{0}^{i}+\delta, x_{0}^{i+1}, \ldots, x_{0}^{n}\right)=a\left(x_{0}\right), \\
& \lim _{\delta \rightarrow 0+} a\left(x_{0}^{1}, x_{0}^{2}, \ldots, x_{0}^{i-1}, x_{0}^{i}-\delta, x_{0}^{i+1}, \ldots, x_{0}^{n}\right)=a\left(x_{0}\right) .
\end{aligned}
$$

It follows from (3.2) and (3.3) that

$$
\lim _{\Delta \rightarrow 0} a\left(x_{0}^{1}, x_{0}^{2}, \ldots, x_{0}^{i-1}, x_{0}^{i}+\Delta, x_{0}^{i+1}, \ldots, x_{0}^{n}\right)=a\left(x_{0}\right) .
$$

This imples that the set-valued map $x^{i} \mapsto a\left(x_{0}^{1}, x_{0}^{2}, \ldots, x_{0}^{i-1}, x^{i}, x_{0}^{i+1}, \ldots, x_{0}^{n}\right)$ is continuous at $x_{0}^{i}$.

Theorem 6. Let $x_{0} \in \mathbb{R}^{n}, f \in \mathbb{R}^{n}$ and let $a(\cdot): \mathbb{R}^{n} \rightarrow \operatorname{comp}\left(\mathbb{R}^{m}\right)$ be a set-valued map. Suppose that the set $\operatorname{graph}_{D} a(\cdot)=\left\{(x, y) \in D \times \mathbb{R}^{m}: y \in a(x)\right\}$ is compact for any compact set $D \subset \mathbb{R}^{n}$. If $D^{*} a\left(x_{0}, y_{0}\right) \mid(f) \neq \varnothing$ for all $y_{0} \in a\left(x_{0}\right)$, then the set-valued map $a(\cdot)$ is continuous at $x_{0}$ in the direction $f$.

Proof. First, we will show that the set-valued map $a(\cdot)$ is upper semi continuous at $x_{0}$ in the direction $f$.

Since the set $\bar{B}\left(x_{0}, 1\right)=\left\{x \in \mathbb{R}^{n}:\left\|x-x_{0}\right\| \leq 1\right\}$ is compact, it follows that the set $\operatorname{graph}_{\bar{B}\left(x_{0}, 1\right)} a(\cdot)$ is compact. Then, we obtain that the set-valued map $a(\cdot): \bar{B}\left(x_{0}, 1\right) \rightarrow$ comp $\left(\mathbb{R}^{m}\right)$ is locally bounded at $x_{0}$ and its graph is closed. From Theorem 2, the setvalued map $a(\cdot): \bar{B}\left(x_{0}, 1\right) \rightarrow \operatorname{comp}\left(\mathbb{R}^{m}\right)$ is upper semi-continuous at $x_{0}$. Hence, $a(\cdot)$ is upper semi-continuous at $x_{0}$ in the direction $f$ and then the set valued map $\delta \rightarrow a\left(x_{0}+\delta f\right)$ is upper semi-continuous on the right at $\delta=0$.

Now we will show that the set-valued map $\delta \mapsto a\left(x_{0}+\delta f\right)$ is lower semi-continuous on the right at $\delta=0$, i. e., we will show that for all $y_{0} \in a\left(x_{0}\right)$ and for all $\left\{\delta_{k}\right\}_{k=1}^{\infty}$ where $\delta_{k} \rightarrow+0$ as $k \rightarrow \infty$, there exists $y_{k} \in a\left(x_{0}+\delta_{k} f\right)$ such that $y_{k} \rightarrow y_{0}$ as $k \rightarrow \infty$.

Take $y_{0} \in a\left(x_{0}\right)$. From the hypothesis of the theorem, $D^{*} a\left(x_{0}, y_{0}\right) \mid(f) \neq \varnothing$. Take $d_{*} \in D^{*} a\left(x_{0}, y_{0}\right) \mid(f)$. From the definition of $D^{*} a\left(x_{0}, y_{0}\right) \mid(f)$, there exists a $\delta_{*}>0$ such that for all $\delta \in\left[0, \delta_{*}\right]$,

$$
y(\delta)=y_{0}+\delta d_{*}+\delta s(\delta) \in a\left(x_{0}+\delta f\right)
$$

where $s(\delta) \rightarrow 0$ as $\delta \rightarrow+0$. For any sequence $\left\{\delta_{k}\right\}_{k=1}^{\infty}$ where $\delta_{k} \rightarrow+0$ as $k \rightarrow \infty$, there exists a $k_{0} \in \mathbb{N}$ such that $\delta_{k} \in\left[0, \delta_{*}\right]$ for all $k \geq k_{0}$. Then for all $k \geq k_{0}$,

$$
\begin{aligned}
y_{k} & =y\left(\delta_{k}\right) \\
& =y_{0}+\delta_{k} d_{*}+\delta_{k} s\left(\delta_{k}\right) \in a\left(x_{0}+\delta_{k} f\right) .
\end{aligned}
$$

Hence, we obtain that $y_{k} \in a\left(x_{0}+\delta_{k} f\right)$ and $y_{k} \rightarrow y_{0}$ as $k \rightarrow \infty$ and hence the set-valued map $\delta \rightarrow a\left(x_{0}+\delta f\right)$ is lower semi-continuous on the right at $\delta=0$.

Consequently, we obtain that the set-valued map $\varphi(\cdot): \mathbb{R} \rightarrow \operatorname{comp}\left(\mathbb{R}^{m}\right), \delta \mapsto$ $\varphi(\delta)=a\left(x_{0}+\delta f\right)$, is upper and lower semi-continuous on the right at $\delta=0$ and then it is continuous on the right. Therefore, $a(\cdot)$ is continuous at $x_{0}$ in the direction $f$. 
Corollary 1. Let $[a, b] \subset \mathbb{R}, x_{0} \in(a, b)$ and let $a(\cdot):[a, b] \rightarrow \operatorname{comp}\left(\mathbb{R}^{m}\right)$ be a set-valued map and let graph $a(\cdot)$ be a compact set. If $D^{*} a\left(x_{0}, y\right) \mid(1) \neq \varnothing$ and $D^{*} a\left(x_{0}, y\right) \mid(-1) \neq \varnothing$ for all $y \in a\left(x_{0}\right)$, then $a(\cdot)$ is continuous at $x_{0}$.

Proof. Since $D^{*} a\left(x_{0}, y\right) \mid(1) \neq \varnothing$ for all $y \in a\left(x_{0}\right)$, by Theorem 6 ,

$$
\lim _{\delta \rightarrow 0+} a\left(x_{0}+\delta\right)=a\left(x_{0}\right)
$$

and since $D^{*} a\left(x_{0}, y\right) \mid(-1) \neq \varnothing$ for all $y \in a\left(x_{0}\right)$, from Theorem 6,

$$
\lim _{\delta \rightarrow 0+} a\left(x_{0}-\delta\right)=a\left(x_{0}\right) .
$$

Then from (3.4) and (3.5),

$$
\lim _{\delta \rightarrow 0} a\left(x_{0}+\delta\right)=a\left(x_{0}\right) .
$$

Hence, $a(\cdot)$ is continuous at $x_{0}$.

Remark 4. The following example shows that Theorem 6 and Corollary 1 are not true when the condition $D^{*} a(x, y) \mid(f) \neq \varnothing$ is replaced by the condition $D a(x, y) \mid$ $(f) \neq \varnothing$.

Example 3. Let us set, for $x \in[-1,1]$,

$$
a(x)= \begin{cases}{[-1,1]} & \text { for } x \in[-1,0], \\ \sin \frac{1}{x} & \text { for } x \in(0,1] .\end{cases}
$$

One can show that the set-valued map $x \mapsto a(x)$ is not continuous at $x_{0}=0$ in the direction $f=1$. However,

$$
\begin{gathered}
\operatorname{Da}(0, y) \mid(1)= \begin{cases}(-\infty, 0] & \text { for } y=1, \\
(-\infty,+\infty) & \text { for } y \in(-1,1), \\
{[0,+\infty)} & \text { for } y=-1,\end{cases} \\
\operatorname{Da}(0, y) \mid(-1)= \begin{cases}(-\infty, 0] & \text { for } y=1, \\
(-\infty,+\infty) & \text { for } y \in(-1,1), \\
{[0,+\infty)} & \text { for } y=-1,\end{cases}
\end{gathered}
$$

and then for all $y \in[-1,1]=a(0), D a(0, y)|(1) \neq \varnothing, D a(0, y)|(-1) \neq \varnothing$. On the other hand,

$$
D^{*} a(0, y) \mid(-1)= \begin{cases}l(-\infty, 0] & \text { for } y=1, \\ (-\infty,+\infty) & \text { for } y \in(-1,1), \\ {[0,+\infty)} & \text { for } y=-1,\end{cases}
$$

and $D^{*} a(0, y) \mid(1)=\varnothing$ for all $y \in[-1,1]$.

We obtain the following corollary from Theorem 6 and Theorem 5 .

Let $f_{i}^{+}=(0,0, \ldots, 0,1,0, \ldots, 0)$ and $f_{i}^{-}=(0,0, \ldots, 0,-1,0, \ldots, 0)$ where $i=$ $1,2, \ldots, n$ and 1 and -1 are $i$ th coordinates of the vectors $f_{i}^{+}$and $f_{i}^{-}$, respectively. 
Corollary 2. Let $a(\cdot): \mathbb{R}^{n} \rightarrow \operatorname{comp}\left(\mathbb{R}^{m}\right)$ be a set valued map. Suppose that $x_{0} \in \mathbb{R}^{n}$ and the set

$$
\operatorname{graph}_{D} a(\cdot)=\left\{(x, y) \in D \times \mathbb{R}^{m}: y \in a(x)\right\}
$$

is compact for any compact set $D \subset \mathbb{R}^{n}$. If $D^{*} a\left(x_{0}, y\right) \mid\left(f_{i}^{+}\right) \neq \varnothing$ and $D^{*} a\left(x_{0}, y\right) \mid$ $\left(f_{i}^{-}\right) \neq \varnothing$ for all $y_{0} \in a\left(x_{0}\right)$ and for all $i=1,2, \ldots, n$, then the set-valued map $x \mapsto a(x)$ is continuous according to all coordinates.

Remark 5. We will show that the converse of Theorem 6 is not true in general.

Example 4. Take the set-valued map $a(\cdot):[-1,1] \rightarrow \operatorname{comp}(\mathbb{R})$,

$$
x \longmapsto a(x)=\{y \in \mathbb{R}: \sqrt[3]{x} \leq y \leq 2\} .
$$

One can show that $a(\cdot)$ is continuous at $x_{0}=0$ and then $a(\cdot)$ is continuous at $x_{0}=0$ in any direction $f$.

We know that $(0,0) \in \operatorname{graph} a(\cdot)$ and

$\operatorname{Da}(0,0) \mid(1)=\left\{v \in \mathbb{R}: \exists \delta_{k}>0\right.$ such that $\delta_{k} \rightarrow 0+$ as $k \rightarrow \infty$,

$$
\left.\exists y_{k} \in a\left(\delta_{k}\right) \ni \lim _{k \rightarrow \infty} y_{k} / \delta_{k}=v\right\}
$$

Take a sequence $\left\{y_{k}\right\}_{k=1}^{\infty}$ such that $y_{k} \in a\left(\delta_{k}\right)$ and $\delta_{k} \rightarrow 0+$ as $k \rightarrow \infty$. Since $y_{k} \in a\left(\delta_{k}\right)$, we have $y_{k} \geq \sqrt[3]{\delta_{k}}$ and then

$$
\lim _{k \rightarrow \infty} \frac{y_{k}}{\delta_{k}} \geq \lim _{k \rightarrow \infty} \frac{\sqrt[3]{\delta_{k}}}{\delta_{k}}=\lim _{k \rightarrow \infty} \frac{1}{\sqrt[3]{\delta_{k}^{2}}} \rightarrow \infty .
$$

Then $D a(0,0) \mid(1)=\varnothing$ and since $D^{*} a(0,0)|(1) \subset D a(0,0)|(1)$, then it follows that

$$
D^{*} a(0,0) \mid(1)=\varnothing .
$$

Remark 6. The hyphothesis of the above theorem does not imply the continuity of $a(\cdot)$.

Example 5. Let us put, for all $(x, y) \in \mathbb{R} \times \mathbb{R}$,

$$
f(x, y)= \begin{cases}1 & \text { if } y=x^{3}, x>0, \\ 0 & \text { in the other cases }\end{cases}
$$

and take the set-valued map $a(\cdot, \cdot): \mathbb{R} \times \mathbb{R} \rightarrow \operatorname{comp}(\mathbb{R}),(x, y) \mapsto a(x, y)=\{f(x, y)\}$. One can show that the function $f(\cdot, \cdot)$ is not continuous at $(0,0)$.

We have

$$
\frac{\partial f(0,0)}{\partial\left(f_{1}, f_{2}\right)}=\lim _{\delta \rightarrow 0+} \frac{f\left(\delta f_{1}, \delta f_{2}\right)-f(0,0)}{\delta}=0
$$

where $f_{1} \neq 0$ or $f_{2} \neq 0$. Then we get

$$
D^{*} a(0,0,0) \mid\left(f_{1}, f_{2}\right)=\left\{\frac{\partial f(0,0)}{\partial\left(f_{1}, f_{2}\right)}\right\}=\{0\} \neq \varnothing .
$$




\section{Conclusion}

The concepts of directional lower and upper sets of set-valued maps and continuity of the set-valued maps are basic concepts in set-valued analysis. In this study, the directional lower and upper derivative sets of the set valued map the values of which have piecewise smooth boundary are suggested and investigated. Moreover, the connection between the directional continuity and the directional derivative sets of set-valued maps is investigated.

\section{REFERENCES}

[1] Aubin, J.-P. and Celuina, A.: Differential Inclusions, Springer, 1984.

[2] Aubin, J.-P.: Differential games: a viability approach, SIAM J. on Control and Optimization, 1990.

[3] Aubin, J.-P. and Frankowska, H.: Set-Valued Analysis, Birkhauser, Boston, 1990.

[4] Blagodatskikh, V. I. and Filippov, A. F., Differential inclusions and optimal control, Proc. Steklov Inst. of Math., 169 (1986), 199-259.

[5] Bouligand, G.: Sur la semi-continuite d'inclusions et quelques sujets connexes, Enseignement Mathematique, 31 (1932), 14-22.

[6] Bouligand, G.: Introduction à la géométrie infinitésimal directe, Gauthier-Villars, Paris, 1932.

[7] Choquet, G.: Convergences, Annales de l'Université de Grenoble, 23 (1947), 55-112.

[8] Clarke, F. H., Ledyayev, Yu. S., Stern, R. J., and Wolenski, P.: Qualitative Properties of Trajectories of Control Systems: a Survey, J. of Dynamical and Control Systems, 1 (1995), 1-48.

[9] Clarke, F. H., Ledyayev, Yu. S., Stern, R. J., and Wolenski, P. R.: Nonsmooth Analysis and Control Theory, Springer, New York, 1998.

[10] Danskin, J. M.: The Theory of Max-Min and Its Application to Weapons Allocation Problems, Springer, New York, 1967.

[11] Demyanov, V. F. and Vasilyev, L. V.: Non-Differentiable Optimization, Nauka, Moscow, 1981 (in Russian).

[12] Demyanov, V. F. and Rubinov, A. M.: Nonsmooth Analysis and Quasidifferential Calculus, Nauka, Moscow, 1990 (in Russian).

[13] Dubovitskis, D. and Miljutin, A. A.: Necessary conditions for a weak extremum in the general problem of optimal control, Nauka, Moscow, 1971 (in Russian).

[14] Frankowska, H.: Inclusions adjointes associées aux trajectoires minimales d'inclusions différentielles, Comptes Rendus de l'Académie des Sciences, Paris, Serie 1, 297 (1983), 461-464.

[15] Guseinov, H. G., Subbotin, A. I., and Ushakov, V. N.: Derivatives for multivalued mappings with applications to game theoretical problems of control, Problems of Control and Information Theory, 14 (1985), 155-167.

[16] Guseinov, Kн. G. and Ushakov, V. N.: Strongly and weakly invariant sets with respect to differential inclusions, Soviet Math. Dokl., 38, No. 3 (1989), 603-605.

[17] Hu, S. and PAPAgeorgiou, N. S.: Handbook of Multivalued Analysis, Vol. I, Kluwer AP, Dordrecht, 1997.

[18] Kuratowski, K., Topologie, Vols. 1, 2, 4th rev. ed., Panstowowe Wyd. Nauk., Warszawa, 1958 (Academic Press, New York, 1966).

[19] Polovinkin, E. S. and Smirnov, G. V.: Differentiation of multivalued mappings and properties of solutions of differential inclusions, Soviet Math. Dokl., 33 (1986), 662-666.

[20] URSESKu, C.: Tangent sets calculus and necessary conditions for extremality, SIAM J. on Contr. Optimiz., 20 (1982), 563-574. 
[21] WARD, Y. A.: The quantification tangent cones, Can. J. Math., 40 (1988), No. 3, 666-694.

\section{Author's address}

\section{Erdal Ekici:}

Department of Mathematics, Canakkale Onsekiz Mart University, Terzioglu Campus, 17020 CaNAKKALE, TURKEY

E-mail address: eekici@comu.edu.tr 